\title{
Steroid injections in pain management: influence on coronavirus disease 2019 vaccines
}

\author{
Sung Man Hong, Yeon Wook Park, and Eun Joo Choi \\ Department of Anesthesiology and Pain Medicine, Seoul National University Bundang Hospital, Seongnam, Korea
}

Received August 18, 2021

Revised November 4, 2021

Accepted November 12, 2021

Handling Editor: Kyung Hoon Kim

\section{Correspondence}

Eun Joo Choi

Department of Anesthesiology and Pain Medicine, Seoul National University Bundang Hospital, 82 Gumi-ro 173beongil, Bundang-gu, Seongnam 13620, Korea

Tel: +82-31-787-7499

Fax: +82-31-787-4063

E-mail: ejchoi@snubh.org
The coronavirus disease 2019 (COVID-19) pandemic, which has been rampant since the end of 2019, has evidently affected pain management in clinical practice. Fortunately, a COVID-19 vaccination program is currently in progress worldwide. There is an ongoing discussion that pain management using steroid injections can decrease COVID-19 vaccine efficacy, although currently there is no direct evidence to support this statement. As such, the feeling of pain in patients is doubled in addition to the co-existing ill-effects of social isolation associated with the pandemic. Thus, in the COVID-19 era, it has become necessary that physicians be able to provide high quality pain management without negatively impacting COVID-19 vaccine efficacy. Steroids can alter the entire process involved in the generation of adaptive immunity after vaccination. The period of hypophysis-pituitary-adrenal axis suppression is known to be 1 to 4 weeks after steroid injection, and although the exact timing for peak efficacy of COVID-19 vaccines is slightly different for each vaccine, the average is approximately 2 weeks. It is suggested to avoid steroid injections for a total of 4 weeks ( 1 week before and after the two vaccine doses) for the doubleshot vaccines, and for 2 weeks in total ( 1 week before and after vaccination) for a single-shot vaccine. This review focuses on the basic concepts of the various CovID-19 vaccines, the effect of steroid injections on vaccine efficacy, and suggestions regarding an appropriate interval between the administration of steroid injections and the COVID-19 vaccine.

Key Words: Adaptive Immunity; Coronavirus; COVID-19; COVID-19 Vaccines; Immunization Programs; Immunosuppression; Injections; Nerve Block; Pain; Pain Management; Pandemics; Social Isolation; Steroids.

\section{INTRODUCTION}

The coronavirus disease 2019 (COVID-19) pandemic which started at the end of 2019 is still going strong and affecting human populations on a large scale globally. At the end of July 2021, there were more than 196 million confirmed cases, and approximately 4.2 million confirmed deaths attributed to COVID-19 worldwide.

The Pfizer-BioNTech COVID 19 vaccine was first ap- proved for use in the United Kingdom in December 2020, and from that point, COVID-19 vaccines were expected to become a game-changer. To date, more than 3.8 billion doses of COVID 19 vaccine have been administered worldwide based on official reports from the World Health Organization [1]. In South Korea, AstraZeneca AZD1222 (February 10, 2021), Pfizer-BioNTech BNT162b2 (March 5, 2021), Johnson \& Johnson Ad26.COV2.S (April 7, 2021), and Moderna mRNA-1273 (May 21, 2021) have been approved (c) This is an open-access article distributed under the terms of the Creative Commons Attribution Non-Commercial License (http://creativecommons.org/licenses/by-nc/4.0/), which permits unrestricted non-commercial use, distribution, and reproduction in any medium, provided the original work is properly cited.

(C) The Korean Pain Society, 2022
Author contributions: Sung Man Hong: Writing/manuscript preparation; Yeon Wook Park: Writing/manuscript preparation; Eun Joo Choi: Study conception. 
by the Ministry of Food and Drug Safety of Korea [2]. The Korean Government and healthcare organizations are trying hard to curb the COVID-19 pandemic by providing fast and widespread access to COVID-19 vaccines.

It is known that the immune response after vaccination is a critical step in reaching sufficient clinical efficacy from the vaccine. However, interventions in pain management, such as the use of steroids, have raised concerns because these may interfere with the immune response of the vaccine. There has been some debate regarding whether steroid injections can affect the efficacy of COVID-19 vaccines or not. In this review, we will be discussing the different opinions addressing this question.

\section{MAIN BODY}

\section{Immunization by newly developed vaccines during the COVID-19 era}

Adaptive immunity allows the body to remember pathogens that have previously invaded it and to develop a fast and strong subsequent immune response to that pathogen. This is the basic working principle behind the various vaccines in use today. Traditionally, inactivated and live attenuated vaccines have been used primarily, but recently, gene recombination techniques have been introduced in the development of vector vaccines or mRNA vaccines. These newly developed vaccines confer immunity via DNA or mRNA, which can express certain surface proteins of the target pathogen. These vaccines differ from traditional vaccines in the method of exposing antigens to the immune system, but the result is the same, that is, the formation of antibodies by the immune system. During the COVID-19 pandemic, numerous vaccines have been developed worldwide [3]. Although these vaccines differ in their platform and immune acquisition process, the basic principle is the same in that they all promote the formation of antibodies against severe acute respiratory syndrome coronavirus 2 (SARS-CoV-2) through an immune response.

There are three types of vaccines according to the method of approach used to generate this immune response [4]. First, vaccines that use the whole virus or bacterium (live attenuated vaccines, inactivated vaccines, and viral vector vaccines). Second, the ones that use only a portion of the virus or bacterium to activate the immune system (protein subunit vaccines). Finally, vaccines that use the genetic material of the virus or bacterium, which allows the formation of specific proteins (mRNA vaccines and DNA vaccines). The following are introductions to various COVID-19 vaccines.

\section{1) Live attenuated vaccines (example: CoviVac)}

These are vaccines with low toxicity, which are produced by subculturing live pathogens in fertilized eggs and animal cells for a long period of time. A live attenuated pathogen is directly injected into the body; therefore, it is possible to induce an immune response with even a small amount of the vaccine with the added advantage of sustained immunity for a long duration. The only disadvantage is that these cannot be used in immunocompromised patients.

\section{2) Inactivated vaccines (examples: BBIBP-CorV and CoronaVac)}

These are a traditional type of vaccine that contain killed virus and use it as an antigen. The injected inactivated virus induces an immune response in the body and promotes the formation of antibodies. Although this classical method is relatively safe and does not require high technical skills, there is a possibility of structural damage to the epitope during the inactivation process.

\section{3) Vectored vaccines (examples: AstraZeneca AZD1222, Johnson \& Johnson's Ad26.CoV2.S, and Sputnik V)}

These vaccines utilize a vector to deliver the target genes of a pathogen into the host cell. In the case of COVID-19, a non-replicating adenovirus vector embedded with the spike protein encoding the DNA of SARS-CoV-2 is used. The vector infects the host cells, resulting in the creation of an antigen. This antigen is then expressed on the host cell surface, resulting in the induction of an immune response [5]. However, there is a risk of generation of an immune response against the vector itself.

\section{4) Protein subunit vaccines (example: NovaVax NVX- COV2373)}

Only a subunit/fragment of the surface protein of the pathogen is extracted for injection by using a genetic recombination method. The injected protein subunit acts as an antigen and induces an immune response. In the case of NovaVax NVX-COV1273, a recombinant protein nanoparticle composed of trimeric spike glycoproteins along with a potent Matrix-Ml adjuvant is used [5].

\section{5) mRNA vaccines (examples: Pfizer-BioNTech BNT162b2} and Moderna mRNA-1273)

This is a newly developed technique of vaccine production using lab-created mRNA. In the case of COVID-19, 
SARS-CoV-2 spike protein encoding mRNA is used. These vaccines deliver mRNA to the cytoplasm, which is then transcribed by ribosomes in the cytoplasm to present spike proteins on the cell surface [5]. Although safety is high and rapid development is possible, transportation and management are difficult due to the instability of RNA and lipid nanoparticles.

\section{6) DNA vaccines (example: INO-4800)}

In this category, the nucleotide sequence corresponding to the antigen is inserted into the plasmid and injected. As with the mRNA vaccines, the host cell directly generates a spike protein and induces an immune response, but these vaccines are more stable and easier to transport and store than the mRNA vaccines. A separate delivery system is not required, and the vaccine is delivered into cells through electroporation after intramuscular injection [3]. The above COVID-19 vaccines are evaluated by their efficacy and effectiveness. The vaccine efficacy is defined by a clinical trial, which is conducted to test the vaccine performance under ideal conditions. For example, if a vaccine is shown to have an efficacy of $70 \%$, it means that $70 \%$ of people who get this vaccine are less likely to contract the disease than those who get a placebo [6,7]. The efficacy of COVID-19 vaccines approved for use in South Korea is in the range of $72 \%-95 \%$ approximately (Table 1 ).

Otherwise, the vaccine effectiveness is determined by how successfully they protect entire communities in the real world. Although clinical trials include people of varied ethnicities and races, these groups cannot sufficiently represent all the people in the real world. The clinical trials of these COVID-19 vaccines excluded or limited the participation of people who are immunocompromised by steroid use. It is possible that the use of steroids may potentially reduce the efficacy or effectiveness of these vaccines.

\section{Effect of steroids on the immune response after vaccination}

Specific adaptive immunity formed after vaccination con- sists of antibody-mediated humoral immunity and cellular immunity (involving B and T lymphocytes, respectively). Adaptive immunity takes from days to weeks to develop fully, but ultimately, the desired effect of the vaccine is achieved by the establishment of immunological memory [8].

Steroids may interfere with vaccine immunity because of their anti-inflammatory and immunosuppressive effects. Exogenous steroids may also influence all stages of the immune response since all immune cells express corticosteroid receptors. Antigen-presenting cells must be able to activate naive $\mathrm{T}$ cells in order to initiate the adaptive immune response, but it is a known fact that steroids disrupt naive $\mathrm{T}$ cell activation by suppressing the ability of antigen presenting cells [9]. Also, T and B cell proliferation, which continues over several weeks to establish adaptive immunity can also be affected by steroids [10-12]. Thus, steroids can alter the entire process involved in the generation of adaptive immunity after vaccination.

\section{1) Effect of systemic steroids on the immune response} after vaccination

Steroids have been used successfully in the treatment of various inflammatory diseases [13]. Questions have been raised as to whether the vaccine-induced antibody formation process is suppressed by steroids or not, and there have been various studies on this topic. One of the studies revealed that antibodies were successfully formed after influenza vaccination in patients receiving steroids for respiratory diseases [14]. Another study reported that the rate of antibody formation after influenza vaccination in patients receiving steroids for rheumatic disease was not different from that of normal patients [15]. Yet another study showed that there was no effect on pneumococcal antibody formation in patients with community aquired pneumonia regardless of dexamethasone treatment. In the light of the above information, it cannot be concluded that steroids reduce the vaccination response.

The Advisory Committee on Immunization Practices recommends that the administration of $20 \mathrm{mg}$ or more of

Table 1. Efficacy and effectiveness of coronavirus disease 2019 (COVID-19) vaccines approved in South Korea

\begin{tabular}{lccc}
\hline \multicolumn{1}{c}{ Vaccines } & Efficacy following first dose & \multicolumn{1}{c}{ Dosage } & Efficacy following second dose \\
\hline AstraZeneca AZD1222 & - & 2 shots, 4 to 12 weeks apart & $76 \%(15 \text { days })^{a}$ \\
Pfizer-BioNTech BNT162b2 & $52 \%(12 \text { days })^{b}$ & 2 shots, 21 days apart & $95 \%(7 \text { days })^{b}$ \\
Moderna mRNA-1273 & - & 2 shots, 28 days apart & $94.1 \%(14 \text { days })^{c}$ \\
Johnson \& Johnson Ad26.COV2.S & - & 1 shot & $72 \%^{\mathrm{d}}$ \\
\hline
\end{tabular}

Information for United Kingdom recipients on COVID-19 vaccine AstraZeneca (Regulation 174), Updated 19 July 2021. https://www.gov.uk/government/publications/regulatory-approval-of-covid-19-vaccine-astrazeneca/information-for-uk-recipients-on-covid-19-vaccine-astrazeneca. ${ }^{b} \mathrm{https} / /$ www. bmj.com/content/371/bmj.m4826. ${ }^{c} \mathrm{https}: / /$ www.cdc.gov/coronavirus/2019-ncov/vaccines/different-vaccines/Moderna.html. ${ }^{\mathrm{d}} \mathrm{https}$ ://www.nytimes. com/2021/02/24/science/johnson-johnson-covid-vaccine.html. 
prednisolone per day continuously over 14 days has an immunosuppressive effect, and caution should be taken in administering live vaccines during this time. Patients who are receiving high-dose steroids are excluded for the clinical trial of vaccines and the use of higher than normal doses of steroids are considered to reduce the vaccination response $[8,16,17]$. In previous reports, pneumococcal vaccinations have been shown to reduce immune responses at doses of $20 \mathrm{mg}$ or more of prednisone per day [18]. There have also been reports that Hepatitis B or influenza vaccination response was delayed in patients on chronic systemic steroids $[19,20]$. Based on previous reports, patients on high dose of systemic steroids have reduced immune response after vaccination, but this has not been demonstrated in all patients.

The timing of vaccination and steroid use may also affect the vaccine response. A recent study reports that dexamethasone premedication reduces strong $\mathrm{T}$ cell immune response as an RNA vaccine-mediated immune effect, in vivo, in human peripheral blood mononuclear cells. Interestingly, immune responses are less impacted when steroids are administered post-vaccination [21]. This means that not only the steroid use itself, but also the timing of steroid use, can be an important factor in determining vaccine efficacy. Although the dose, total period of usage, timing of steroid use, and the individual immune status of patients on systemic steroids are expected to affect vaccine efficacy, no studies have analyzed these aspects in detail to date.

2) Effect of locally injected steroids on the immune response after vaccination

As with systemic steroids administered intravenously or orally, locally injected steroids can be absorbed systemically and may exhibit systemic effects [22,23]. Even topical steroids can exhibit systemic effects to the extent of suppressing cellular immunity when used for a long period [24]. A previous study revealed that the influenza infection rate was higher in flu-vaccinated patients who received intra-articular steroid injections compared to a similar cohort that did not receive a steroid injection [25]. However, the available evidence is insufficient to conclusively state that locally injected steroids reduce vaccine efficacy, and it is also unclear whether a single steroid injection can have a similar systemic effect or not.

Some researchers suggested that adaptive immune response and establishment of immunological memory may be affected by the timing of hypothalamic-pituitaryadrenal (HPA) axis suppression following locally administered steroid injections. The HPA axis is suppressed for 1 to 4 weeks after a single intra-articular steroid injection, and in some cases this duration may be longer [26-28]. HPA axis suppression has also been reported following epidural steroid injection. One study showed that more than $80 \%$ of patients who were administered an epidural injection of $80 \mathrm{mg}$ of methylprednisolone experienced HPA axis suppression for a duration of 1 to 4 weeks [29]. Another study showed that adrenocorticosteroid hormone and cortisol production were decreased for 1 and 4 weeks respectively after an epidural injection of $80 \mathrm{mg}$ of triamcinolone [30].

These results suggest a relationship between immunosuppression following epidural steroid injection and decreased vaccination response. However, no studies have investigated the direct relationship between the two. Therefore, it is not clear how the type, dose, and duration (single injection or numbers of injections) of steroids used for epidural and musculoskeletal injections affect vaccination response.

\section{Does steroid injection for pain management really reduce the efficacy of the COVID-19 vaccine?}

To date, there is no direct evidence that single steroid injection affects vaccine efficacy. Actually, although several newly developed COVID-19 vaccines are undergoing rigorous clinical trials, there has been no study exploring the relationship between epidural or musculoskeletal steroid injections for pain management and COVID-19 vaccine response or efficacy so far. Therefore, when considering the immunosuppressive effect of steroids, it is important to conservatively consider whether to administer steroid injection before and after vaccination.

There are recommendations that COVID-19 vaccination should be avoided during the period of suppression of the HPA axis and cortisol secretion following steroid injection $[8,31]$. Lee et al. [8] recommended setting up a COVID-19 vaccination plan (mRNA vaccines; Pfizer-BioNTech, and Moderna vaccines) considering both the period of suppression of the HPA axis after steroid injection and the timing for peak efficacy of the COVID 19 vaccine. The period of HPA axis suppression is known to be 1 to 4 weeks after steroid injection and although the exact timing for peak efficacy of COVID-19 vaccines is slightly different for each vaccine, the average is approximately 2 weeks (Table 1).

Based on these facts Lee et al. [8] advised that physicians should deliver a steroid injection no less than 2 weeks prior to vaccination and no less than 1 week after vaccination. Other researchers have advised that patients should be informed about the immunosuppressive risks of steroids and to avoid steroid injections starting from 1 week prior to the first dose of the vaccine and up to 1 week after the second dose, for the mRNA vaccines, as these are the major type 
of COVID-19 vaccines in use currently [31,32]. The interval of 1 week before and after vaccination is thought to set the minimum period during which the HPA axis is suppressed even though there are some patients in which it is suppressed for a longer period. This is thought to be intended to minimize the period of inhibition from steroid injection because the effect of steroid injection on vaccine efficacy is still not clear.

Studies have also recommended that dexamethasone or betamethasone be used in steroid injection, if necessary, because epidural injection with dexamethasone or betamethasone did not lead to a decrease in cortisol production when compared to injection of local anesthetics [33]. However, the effect of epidural injection with nonparticulate steroid on the HPA axis is still uncertain and further clinical studies are needed.

On the contrary, the American Society of Pain and Neuroscience published counter recommendations and guidelines. They insisted that there are no data that suggest epidural steroid injections should be postponed or avoided due to COVID-19 vaccination. Also, there has been no direct evidence to prove a decrease of COVID-19 vaccine efficacy when steroid injection is combined with the vaccine. They recommended that clinicians should consider the timing of steroid injection in correlation with COVID-19 vaccine administration taking into consideration individual patient's risk. These factors include the urgency of the situation and the underlying diseases and medical conditions affecting the immune status of the patient [32].

In immunocompromised patients, who sometimes require steroid supplementation, if symptoms worsen after vaccination, treatment may be more important than vaccine efficacy, and steroid supplementation should not be delayed. An exacerbation of their underlying disease could potentially raise the risk of acquiring COVID-19 [34].

It is evident that there is rising concern about COVID-19 vaccine efficacy being affected by steroid administration, despite the fact that, based on the data so far, direct evidence is lacking, except in the special cases mentioned above.

\section{Clinical considerations and recommendations for steroid injections for pain management during the COVID-19 era}

The Korean Pain Society (KPS) has recommended that steroid injection should be avoided from 1 week before to 1 week after the vaccination, and if necessary, dexamethasone or betamethasone should be used [35]. Also, they suggested selective pain intervention in urgent or emergency cases in the COVID-19 era.

The American Society of Interventional Pain Physicians has also suggested considering the degree of urgency of the procedure [36]. Physicians can decide the type of pain management intervention associated with steroid injections according to the status and severity of the pain. Table 2 shows detailed interventions based on the categories of urgency. The recommended categories have been decided according to the various circumstances encountered in day-to-day medical practice.

Based on the guidelines of the KPS [35], the following recommendations are to be followed with regard to pain intervention with steroids and COVID-19 vaccination.

Table 2. General guidelines for the management of patients with pain during the coronavirus disease 2019 (COVID-19) pandemic [36]

\begin{tabular}{cll}
\hline Urgency & \multicolumn{1}{c}{ Examples } & \multicolumn{1}{c}{ Intervention examples } \\
\hline Emergency & Removal of implanted devices due to complication & Removal of devices \\
& SCS implantation or removal after SCS trial & Implantation or removal of SCS \\
& SCS electrodes migration with neurological deficit & Repositioning of SCS leads \\
ITDDS drug stock finished & Pump refill \\
& Persistent and severe PDPH & Epidural blood patch \\
Neurolysis for severe cancer pain & Epidural steroids injection \\
& HIVD with acute neurologic deficit & Vertebroplasty or kyphoplasty \\
& Compression fracture of spine with severe pain & Battery change \\
& SCS battery dysfunction & Sympathetic nerve block \\
& Sympathetic nerve block in early stage of CRPS & Radiofrequency ablation \\
& Recurred pain after previous radiofrequency ablation & SI joint block \\
& Acute SI joint pain & Peripheral nerve block \\
& Peripheral nerve entrapment with neurologic deficit & MBB, discography, etc. \\
Diagnostic intervention & Epidural steroid injection \\
& Epidural steroid injection for chronic pain control & TPI, intra-articular injection, etc. \\
\hline
\end{tabular}

SCS: spinal cord stimulator, ITDDS: intrathecal drug delivery system, PDPH: post-dural puncture headache, HIVD: herniated intervertebral disc, CRPS: complex regional pain syndrome, SI: sacroiliac, MBB: medial branch block, TPI: trigger point injection.

Adapted from the article of Gharibo et al. (Pain Physician 2020; 23(4S): S183-204) [36]. 


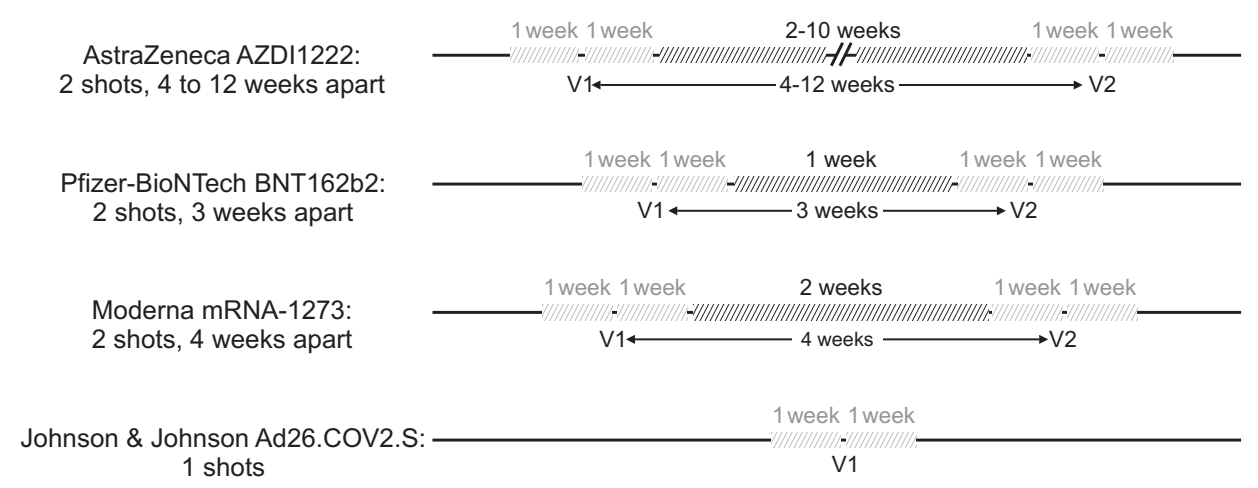

Fig. 1. Suitable time interval between pain management using steroids and coronavirus disease 2019 (COVID-19) vaccination, before and after the vaccination. In a 2 shot vaccine (AstraZeneca AZD1222, Pfizer-BioNTech BNT162b2, Moderna mRNA-1273), it is desirable to avoid steroid injections for a total of 4 weeks ( 1 week before and after the two vaccine doses), and in a single shot vaccine (Johnson \& Johnson Ad26.COV2.S), this duration is 2 weeks in total (1 week before and after vaccination).

1) Clinicians can deliver a steroid injection no less than 1 week prior to and 1 week after vaccination. However, if the patient is immunocompromised or a chronic steroid user, physicians should consider a steroid injection no less than 2 weeks or more prior to and 1 week or more after vaccination. In Fig. 1, the timing of pain intervention with steroids is represented with focus on COVID-19 vaccines approved in South Korea.

(1) For the vaccines that are administered as 2 shots (AstraZeneca AZD1222, Pfizer-BioNTech BNT162b2, and Moderna mRNA-1273), it is desirable to avoid steroid injections for a total of 4 weeks, and for the single shot vaccine (Johnson \& Johnson Ad26.COV2.S), this duration must be 2 weeks in total (before and after vaccination).

(2) In case of AstraZeneca AZD1222, steroid injections can be given during the 2-10-week interval between the first and the second shots.

(3) In case of the Pfizer-BioNTech BNT162b2, steroid injection administration can be performed in the 1-weekinterval between the first and the second shots, but this is not advisable as this period is very short.

(4) For Moderna mRNA-1273, steroid injections are allowed in the 2-week gap between the first and second shots.

2) For pain intervention with steroids, dexamethasone and betamethasone are recommended.

3) If pain intervention with steroids is to be performed during a period in which the steroid injection is prohibited, the final decision should be based on the situation (emergency or urgent) of the patient upon presentation.

\section{CONCLUSIONS}

Although there is no evidence in support of the fact that steroid injections for pain management can affect the efficacy of the COVID-19 vaccines, clinicians should consider every individual's risk and modify the management plan accordingly. If a steroid injection has to be avoided for a certain period of time to maintain vaccine efficacy, this period should be kept to the minimum. In the light of the above discussion, it is evident that clinicians must pay increased attention to pain management in the COVID-19 pandemic era.

\section{CONFLICT OF INTEREST}

No potential conflict of interest relevant to this article was reported.

\section{FUNDING}

No funding to declare.

\section{ORCID}

Sung Man Hong, https://orcid.org/0000-0002-3375-5421

Yeon Wook Park, https://orcid.org/0000-0001-6202-1673

Eun Joo Choi, https://orcid.org/0000-0002-7002-3932

\section{REFERENCES}

1. World Health Organization. WHO Corona virus (COVID-19) dashboard [Internet]. Geneva: World Health Organization; 
2021. Available at: https://covid19.who.int/.

2. Ministry of Food and Drug Safety of Korea. Information of Corona virus (COVID-19) vaccines in Korea [Internet]. Cheongju: Ministry of Food and Drug Safety of Korea; 2021. Available at: https://www.mfds.go.kr/vaccine_covid19.jsp.

3. Tregoning JS, Brown ES, Cheeseman HM, Flight KE, Higham SL, Lemm NM, et al. Vaccines for COVID-19. Clin Exp Immunol 2020; 202: 162-92.

4. Nagy A, Alhatlani B. An overview of current COVID-19 vaccine platforms. Comput Struct Biotechnol J 2021; 19: 2508-17.

5. Yoo JH. What we do know and do not yet know about COVID-19 vaccines as of the beginning of the year 2021. J Korean Med Sci 2021; 36: e54.

6. Lipsitch M, Kahn R. Interpreting vaccine efficacy trial results for infection and transmission. Vaccine 2021; 39: 4082-8.

7. Polack FP, Thomas SJ, Kitchin N, Absalon J, Gurtman A, Lockhart S, et al. Safety and efficacy of the BNT162b2 mRNA Covid-19 vaccine. N Engl J Med 2020; 383: 2603-15.

8. Lee H, Punt JA, Miller DC, Nagpal A, Smith CC, Sayeed Y, et al. Do corticosteroid injections for the treatment of pain influence the efficacy of mRNA COVID-19 vaccines? Pain Med 2021; 22: 994-1000.

9. Strehl C, Ehlers L, Gaber T, Buttgereit F. Glucocorticoids-allrounders tackling the versatile players of the immune system. Front Immunol 2019; 10: 1744.

10. Akondy RS, Fitch M, Edupuganti S, Yang S, Kissick HT, Li $\mathrm{KW}$, et al. Origin and differentiation of human memory CD8 T cells after vaccination. Nature 2017; 552: 362-7.

11. Lindgren G, Ols S, Liang F, Thompson EA, Lin A, Hellgren $\mathrm{F}$, et al. Induction of robust $\mathrm{B}$ cell responses after influenza mRNA vaccination is accompanied by circulating hemagglutinin-specific ICOS+ PD-1+ CXCR3+ T follicular helper cells. Front Immunol 2017; 8: 1539.

12. Hartley GE, Edwards ESJ, Aui PM, Varese N, Stojanovic S, McMahon J, et al. Rapid generation of durable B cell memory to SARS-CoV-2 spike and nucleocapsid proteins in COVID-19 and convalescence. Sci Immunol 2020; 5: eabf8891.

13. Ingawale DK, Mandlik SK. New insights into the novel antiinflammatory mode of action of glucocorticoids. Immunopharmacol Immunotoxicol 2020; 42: 59-73.

14. Kubiet MA, Gonzalez-Rothi RJ, Cottey R, Bender BS. Serum antibody response to influenza vaccine in pulmonary patients receiving corticosteroids. Chest 1996; 110: 367-70.

15. Herron A, Dettleff G, Hixon B, Brandwin L, Ortbals D, Hornick $\mathrm{R}$, et al. Influenza vaccination in patients with rheumatic diseases. Safety and efficacy. JAMA 1979; 242: 53-6.

16. Coutinho AE, Chapman KE. The anti-inflammatory and immunosuppressive effects of glucocorticoids, recent developments and mechanistic insights. Mol Cell Endocrinol 2011; 335: 2-13.

17. Kroger A, Bahta L, Hunter P. General best practice guidelines for immunization. Best practices guidance of the Advisory
Committee on Immunization Practices (ACIP) [Internet]. Atlanta (GA): Centers for Disease Control and Prevention; 2021. Available at: https://www.cdc.gov/vaccines/hcp/acip-recs/ general-recs/index.html.

18. Fischer L, Gerstel PF, Poncet A, Siegrist CA, Laffitte E, Gabay $\mathrm{C}$, et al. Pneumococcal polysaccharide vaccination in adults undergoing immunosuppressive treatment for inflammatory diseases--a longitudinal study. Arthritis Res Ther 2015; 17: 151.

19. Yıldız N, Sever L, Kasapçopur Ö, Çullu F, Arısoy N, Çalışkan S. Hepatitis B virus vaccination in children with steroid sensitive nephrotic syndrome: immunogenicity and safety? Vaccine 2013; 31: 3309-12.

20. Hottinger AF, George AC, Bel M, Favet L, Combescure C, Meier S, et al. A prospective study of the factors shaping antibody responses to the AS03-adjuvanted influenza A/H1N1 vaccine in cancer outpatients. Oncologist 2012; 17: 436-45.

21. Vormehr M, Lehar S, Kranz LM, Tahtinen S, Oei Y, Javinal V, et al. Dexamethasone premedication suppresses vaccineinduced immune responses against cancer. Oncoimmunology 2020; 9: 1758004.

22. Stout A, Friedly J, Standaert CJ. Systemic absorption and side effects of locally injected glucocorticoids. PM R 2019; 11: 40919.

23. Kang WY, Lee JW, Lee E, Kang Y, Ahn JM, Kang HS. Systemic effects of fluoroscopically guided epidural steroid injection with dexamethasone. Korean J Pain 2019; 32: 178-86.

24. Chucair-Elliott AJ, Carr MM, Carr DJJ. Long-term consequences of topical dexamethasone treatment during acute corneal HSV-1 infection on the immune system. J Leukoc Biol 2017; 101: 1253-61.

25. Sytsma TT, Greenlund LK, Greenlund LS. Joint corticosteroid injection associated with increased influenza risk. Mayo Clin Proc Innov Qual Outcomes 2018; 2: 194-8.

26. Habib GS. Systemic effects of intra-articular corticosteroids. Clin Rheumatol 2009; 28: 749-56.

27. Miller DC, Patel J, Gill J, Mattie R, Saffarian M, Schneider BJ, et al. Corticosteroid injections and COVID-19 infection risk. Pain Med 2020; 21: 1703-6.

28. Weitoft T, Rönnblom L. Glucocorticoid resorption and influence on the hypothalamic-pituitary-adrenal axis after intra-articular treatment of the knee in resting and mobile patients. Ann Rheum Dis 2006; 65: 955-7.

29. Abdul AJ, Ghai B, Bansal D, Sachdeva N, Bhansali A, Dhatt SS. Hypothalamic pituitary adrenocortical axis suppression following a single epidural injection of methylprednisolone acetate. Pain Physician 2017; 20: E991-1001.

30. Iranmanesh A, Gullapalli D, Singh R, Veldhuis JD. Hypothalamo-pituitary-adrenal axis after a single epidural triamcinolone injection. Endocrine 2017; 57: 308-13.

31. Brill S, Hochberg U, Goor-Aryeh I. Neuro-axial steroid injection in pain management and COVID-19 vaccine. Eur J Pain 
2021; 25: 945-6.

32. Chakravarthy K, Strand N, Frosch A, Sayed D, Narra LR, Chaturvedi $\mathrm{R}$, et al. Recommendations and guidance for steroid injection therapy and COVID-19 vaccine administration from the American Society of Pain and Neuroscience (ASPN). J Pain Res 2021; 14: 623-9.

33. Friedly JL, Comstock BA, Heagerty PJ, Bauer Z, Rothman MS, Suri $P$, et al. Systemic effects of epidural steroid injections for spinal stenosis. Pain 2018; 159: 876-83.

34. Hasan SS, Capstick T, Zaidi STR, Kow CS, Merchant HA. Use of corticosteroids in asthma and COPD patients with or without COVID-19. Respir Med 2020; 170: 106045.
35. The Korean Pain Society. General guidelines for the management of patients with pain during the COVID-19 global pandemic era [Internet]. Seoul: The Korean Pain Society; 2021. Available at: http://www.painfree.or.kr/board/view. html?code=notify\&num $=10567$.

36. Gharibo C, Sharma A, Soin A, Shah S, Diwan S, Buenaventura $\mathrm{R}$, et al. Triaging interventional pain procedures during COVID-19 or related elective surgery restrictions: evidenceinformed guidance from the American Society of Interventional Pain Physicians (ASIPP). Pain Physician 2020; 23(4S): S183-204. 